\title{
A Comparative evaluation of the Efficacay of two oral medication on post operative pain following single visit root canal therapy - An in vivo study
}

\author{
KrishnaprasadaL ,Jayanth M Nambiar \\ (Department of conservative dentistry \&Endodontics, KVG Dentalcollege\& Hospital) \\ (Department of conservative dentistry \&Endodontics, KVG Dentalcollege\& Hospital)
}

\begin{abstract}
AIM : To compare the effects of single dose of two oral medication following single visit root canal therapy in teeth with irreversible pulpitis

MATERIALS AND METHODS: 30 patients who reported to KVG dental college sullia, seeking treatment on which single visit RCT was performed. In this double blind study anterior or premolar teeth with irreversible pulpitis without any signs and symptoms of irreversible pulpitis were selected divided into 3 groups randomly. A control group without any medication and two groups receiving a single dose of E-90 and Movon-p respectively immediately after the root canal therapy the intensity of pain was recorded on a 10 point VAS for upto $24 \mathrm{hr}$ post operatively.

RESULTS :E-90 Etoricoxib-90 (group 1) was found to be clinically significant at 10\% more compared to the other two groups in the reduction of post operative pain. (Movon-p) wasmore effective compared to the control group where no medication was given

CONCLUSION: A single dose of E-90 considerably reduced thepost operative pain compared to the other two groups taken immediately after the after the RCT of teeth with irreversible pulpitis.

Keywords:Irreversible pulpitis, single visit root canal therapy, postoperative pain,analgesics,periapical lesion.
\end{abstract}

\section{Introduction}

Pain is an unpleasant sensation that can significantly interfere with the person's quality of life and general functioning . The prevalence of post operative pain has been reported to be significantly higher associated with single visit than with multiple visit root canal treatment .The prevalence of post operative pain following root canal treatment has been reported to be $3-58 \%$.

Studies regarding post-obturation pain levels that required analgesics have reported significantly higher prevalence associated with single-visit than with multiple-visit treatment. Non Steroidal Anti-inflammatory drugs (NSAIDS) are the most common analgesics in ambulatory patients. Some drugs can prevent the production of inflammatory mediators involved in producing pain, such as prostaglandins owing to pulpal inflammation and necrosis. NASAIDS are widely available, and they have been reported to be effective in managing endodontic pain . Non narcoticanalgesics ,NSAIDS and acetaminophen can all produce analgesia; therefore, they can be effectivefor managing pulp and periradicular pain . [1]

Several double blind , placebo - controlled trials in patients with endodontic pain have been carried out with NASAIDS namely ibuprofen and it has shown good efficacy and safety profiles. Studies have shown that a suitable method for controlling moderate to severe pain is to combine two or more drugs ;Movon $-\mathrm{P}$ a combination of aceclofenac and paracetamolAceclofenac is a phenylacetic acid derivative that inhibits synthesis of the inflammatory cytokines interleukin- $1 \mathrm{~b}$ and tumour necrosis factor, inhibits prostaglandin E2 production. Paracetamol has analgesic and antipyretic action with weak anti-inflammatory activity. It produces analgesia by increasing pain threshold and antipyresis by acting on the hypothalamic heat-regulating centre. E-(90) Etoricoxib selectively inhibits isoform 2 ofcyclo-oxigenase enzyme (COX-2).[2]

This reduces the generation of prostaglandins (PGs) from arachidonic acid. Among the different functions exerted by PGs, their role in the inflammation cascade should be highlighted. Since no studies have been done using this two oral medication hence the purpose of the study is to compare effects of single dose of two oral medication following single visit root canal therapy in teeth with irreversible pulpitis.

\section{Material And Methods:}

After explanation of the treatment procedures, tooth was anaesthetized. The tooth was considered anaesthetized when two consecutive negative responses to the electric pulp testing was achieved.

All the treatment were performed under rubber dam isolation. An access cavity was prepared,and the working length was determined $1 \mathrm{~mm}$ short of the apex with a size 15 or $20 \mathrm{k}$ file, confirmed with 
Periapicalradiographs. The apical part of each root canal was prepared with the step back technique in a circumferential manner.Normal saline was used as irrigant solution between instrumentation of canals and then dried with paper points, and obturated using lateral condensation technique using zinc oxide eugenol sealer .Each patient's tablet was inserted into a sealed, coded packet by a trained person who was blinded to the drugs , and the packet remained sealed until it was given to the patient.

The visual analogue scale was used to find the intensity of pain at $6 \mathrm{~h}, 12 \mathrm{~h}$ and $24 \mathrm{~h}$ respectively.

$\begin{array}{llllllll}\text { S1. } & & & & & & & \\ \text { No. } & \text { Age } & \text { sex } & 6 \mathrm{~h} & 12 \mathrm{~h} & 24 \mathrm{~h} & \text { group } & \\ 1 & 43 & 2 & 0 & 0 & 0 & 1 & \\ 2 & 22 & 1 & 1 & 0 & 0 & 1 & \\ 3 & 36 & 1 & 2 & 0 & 0 & 1 & \\ 4 & 30 & 1 & 1 & 0 & 0 & 2 & \\ 5 & 21 & 1 & 1 & 0 & 0 & 2 & \\ 6 & 47 & 1 & 0 & 1 & 0 & 2 & \\ 7 & 50 & 2 & 2 & 1 & 0 & 2 & \\ 8 & 21 & 1 & 0 & 0 & 0 & 1 & \\ 9 & 46 & 2 & 0 & 0 & 0 & 3 & \\ 10 & 41 & 2 & 1 & 0 & 0 & 3 & \\ 11 & 19 & 1 & 0 & 0 & 0 & 3 & \\ 12 & 49 & 2 & 2 & 2 & 1 & 3 & \\ 13 & 40 & 2 & 2 & 1 & 0 & 3 & \\ 14 & 40 & 1 & 1 & 0 & 0 & 1 & \\ 15 & 20 & 2 & 1 & 0 & 0 & 2 & \\ 16 & 20 & 1 & 0 & 0 & 0 & 3 & \\ 17 & 28 & 2 & 0 & 0 & 0 & 1 & \\ 18 & 20 & 1 & 0 & 0 & 0 & 1 & \\ 19 & 44 & 2 & 2 & 2 & 0 & 3 & \\ 20 & 19 & 1 & 1 & 0 & 0 & 3 & \\ 21 & 32 & 2 & 0 & 0 & 0 & 2 & \\ 22 & 20 & 1 & 1 & 0 & 0 & 2 & \\ 23 & 18 & 2 & 2 & 0 & 0 & 2 & \\ 24 & 26 & 1 & 0 & 0 & 0 & 1 & \\ 25 & 35 & 2 & 2 & 0 & 0 & 3 & \\ 26 & 28 & 1 & 0 & 0 & 0 & 1 & \\ 27 & 42 & 2 & 1 & 0 & 0 & 2 & \\ 28 & 14 & 1 & 0 & 0 & 0 & 1 & \\ 29 & 24 & 1 & 0 & 0 & 0 & 3 & \\ 30 & 29 & 1 & 0 & 0 & 0 & 2 & \end{array}$

$\mathrm{T}$ test and Kruskalwallis test were done for statistical analysis.

\section{T-Test}

Group Statistics

\begin{tabular}{|c|c|c|c|c|c|}
\hline & GROUP & $\mathrm{N}$ & Mean & Std. Deviation & $\begin{array}{l}\text { Std. Error } \\
\text { Mean }\end{array}$ \\
\hline \multirow[b]{2}{*}{ MEAN } & 1 & 10 & .1333 & .23307 & .07370 \\
\hline & 2 & 10 & .3667 & .29187 & .09230 \\
\hline
\end{tabular}

\section{Independent Samples Test}

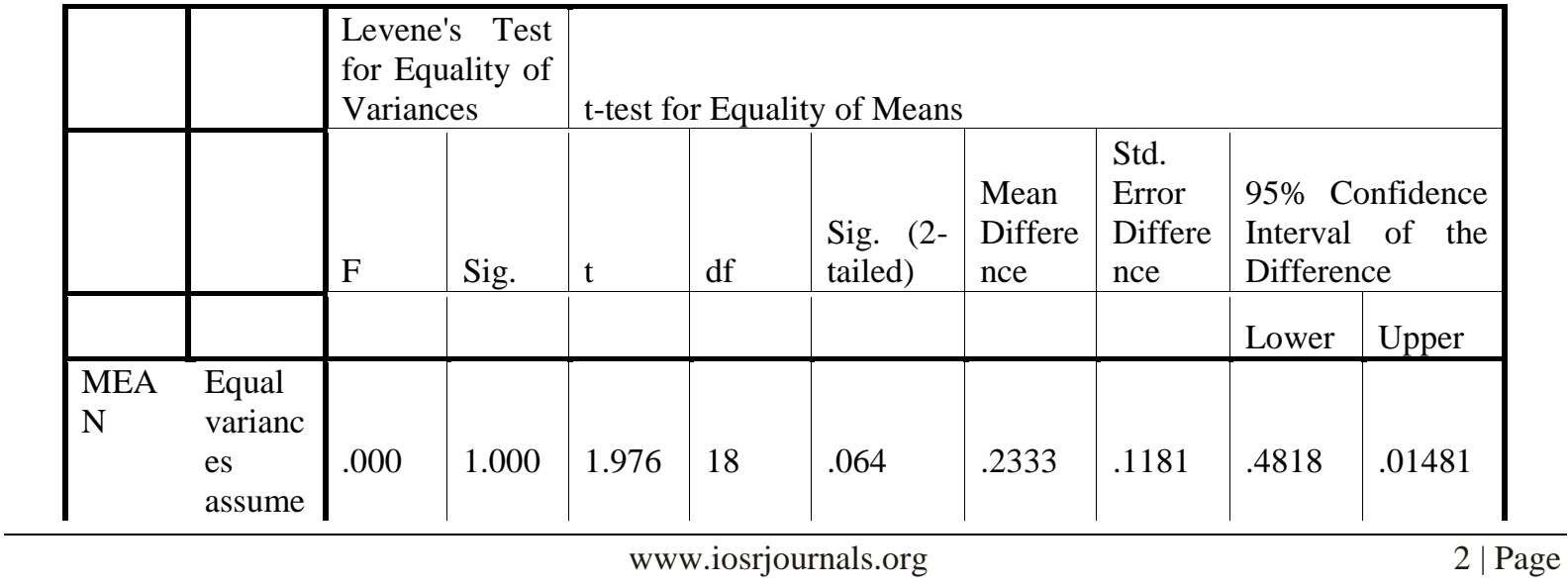




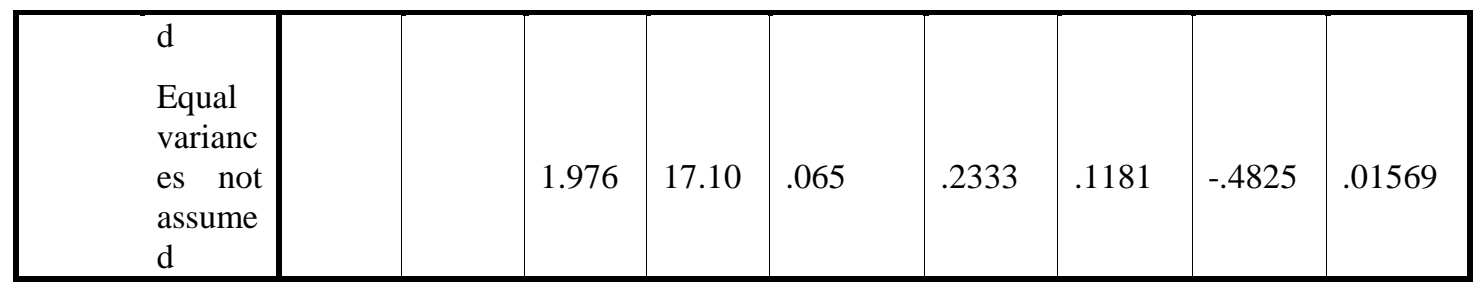

T-Test

Group Statistics

\begin{tabular}{|l|l|l|l|l|l|}
\hline & & & & & Std. Error \\
& GROUP & $\mathrm{N}$ & Mean & Std. Deviation & Mean \\
\hline \multirow{2}{*}{ MEAN } & 1 & 10 & .1333 & .23307 & .07370 \\
& 3 & 10 & .5333 & .61262 & .19373 \\
\hline
\end{tabular}

The statistics was formulated using kruskal - wallisand ' $t$ ' test

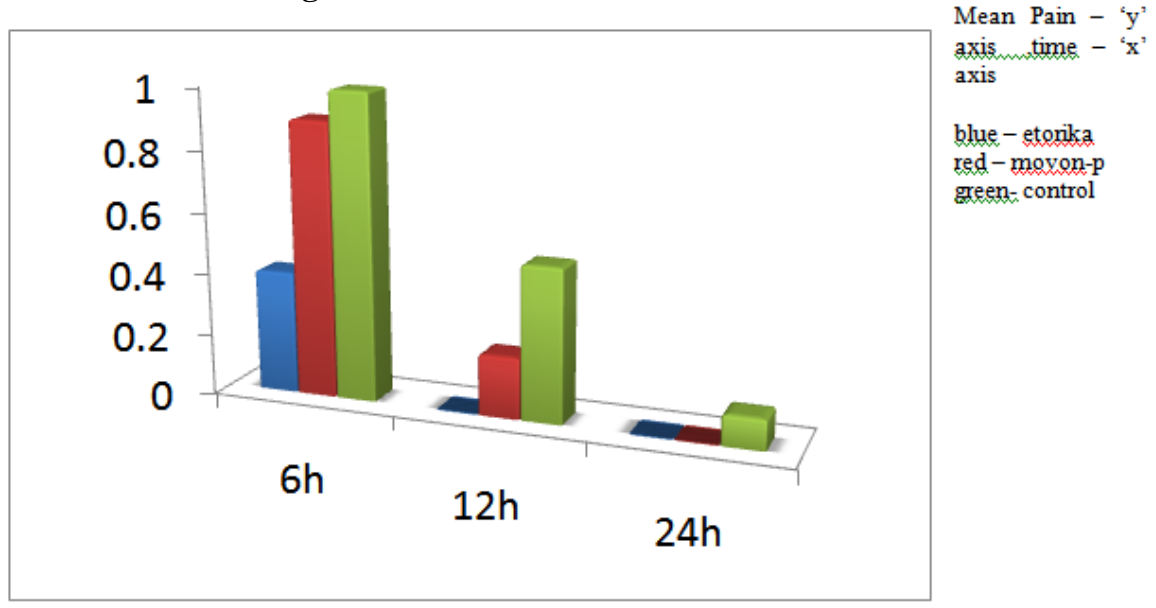

III. Results:

E-90 was found to beclinically significant at $10 \%$ using the Kruskalwallis and ' $T$ ' test. E-90 significantly reduced the post operative pain compared to movon-p, a combination of aceclofenac and paracetamolfollowing single visit root canal therapy .

\section{Discussion}

This double blind study compared the analgesic effects of two different drugs with control . Only patients with irreversible pulpitis without any periapical lesion was selected because of the greatest predictor of post operative pain . Double blind drug administration and elimination or matching of other variables can lead to increased internal validity and more precise results. Patient cooperation and relying on their answers to a questionnaire are other potential problems associated with these types of studies . Most patients experience pain in the first $24 \mathrm{~h}$ after root canal treatment and therefore, this longer period of assessment were chosen even though the drugs tested would not be expected to provide ongoing analgesia for this entire period owing to their relatively short plasma half- lives, which is much less than $24 \mathrm{~h}$.

To evaluate just the effect of the drug and achieve better control of the interfering factors, normal saline was used as the root canal irrigant instead of sodium hypochlorite .

Patients with no history of taking analgesics in the pevious $12 \mathrm{~h}$ or other drugs prior to presenting for treatment were selected to eliminate the interfacing effects of other agents .

Nonsteroidalantiinflammatory drugs (NSAIDs), including selective cyclooxygenase (COX)-2 inhibitors, have come to play an important role in the pharmacologic management of arthritis and pain. Clinical trials have established the efficacy of etoricoxib in osteoarthritis, rheumatoid arthritis, acute gouty arthritis, ankylosing spondylitis, low back pain, acute postoperative pain, and primary dysmenorrhea. Comparative studies indicate at least similar efficacy with etoricoxib versus traditional NSAIDs. Etoricoxib was generally well tolerated in these studies with no new safety findings during long-term administration. The gastrointestinal, renovascular, and cardiovascular tolerability profiles of etoricoxib have been evaluated in large patient datasets. The available data suggest that etoricoxib is an efficacious alternative in the management of arthritis and pain, 
with the potential advantages of convenient once-daily administration and superior gastrointestinal tolerability compared with traditional NSAIDs.[1]

In vitro, etoricoxib exhibits a greater selectivity for COX-2 over COX-1 compared with the COX-2 inhibitors rofecoxib, valdecoxib, and celecoxib.3Etoricoxib binds competitively to COX-2 with 1:1 stoichiometry in a reversible, noncovalent manner. [3]

In human whole blood assays, etoricoxib inhibited COX-2 with an IC50 of $1.1 \pm 0.1 \mu \mathrm{M}$, compared with an IC50 of $116 \pm 8 \mu \mathrm{M}$ for COX-1, representing 106-fold selectivity for COX-2 over COX-1 . No inhibitory effect was observed against a wide range of other receptors and enzymes evaluated. Selective COX-2 inhibition was also observed in ex vivo blood samples from healthy human volunteers who received etoricoxib at various therapeutic and supratherapeuticdoses .[3]

Etoricoxib produced markedly less interference with the cardioprotective COX-1-mediated antiplatelet activity of low-dose aspirin in vitro than other NSAIDs including (in ascending order of aspirin antagonism) rofecoxib, valdecoxib, celecoxib, and ibuprofen, reflecting the lower affinity of etoricoxib for COX-1.[4]

These findings are consistent with results from clinical studies in which ibuprofen, but not rofecoxiboretoricoxib , antagonized aspirin antiplatelet activity.[5]

Etoricoxib showed potent, dose-dependent efficacy similar to other NSAIDs in animal models of acute inflammation, hyperalgesia, pyresis, and chronic adjuvant-induced arthritis.Preclinical and clinical data were consistent with the gastrointestinal tolerability of selective COX-2 inhibition; no effects on gastrointestinal integrity were observed in animal models as measured by urinary or fecal excretion of 51creatinineethylenediamine tetra acetic acid (51Cr-EDTA), 7 an inert compound that is not taken up by extravascular tissue after its absorption from the gastrointestinal tract, but is completely excreted by the kidney .2 Inhibition of prostaglandin synthesis in the gastric mucosa was not significantly different from that of placebo in human volunteers .[4]

Aceclofenac (ACF) , [(\{2-[(2,6-dichlorophenyl)amino]phenyl $\}$ acetyl)oxy $]$ acetic acid , has analgesic properties and a good tolerability profile in a variety of painful conditions. It is used for treatment of rheumatic disorders and soft-tissue injuries. ACF inhibits the enzyme cyclooxygenase and thus exerts its anti-inflammatory activity by inhibition of prostaglandin synthesis. This effect seems to be correlated with the appearance of acute protocolitis associated with nonsteroidal anti-inflammatory drug therapy .[5]

Paracetamol (PCM), $N$-(4-hydroxyphenyl)acetamide, is a non-opiate, non-salicylate, centrally and peripherally acting analgesic agent. It has only weak anti-inflammatory effects. The most commonly consumed daily dose, $1000 \mathrm{mg}$, results in roughly $50 \%$ inhibition of both COX-1 and COX-2 in whole blood assays ex vivo in healthy volunteers. It has been suggested that COX inhibition might be disproportionately pronounced in the brain, explaining its antipyretic efficacy and its direct activity on the centre for the body temperature regulation in the hypothalamus. [6]

The results demonstrated that a single oral dose of Etorica-90 capsule immediately after root canal preparation reduced the intensity of post operative pain effectively and a combination of aceclofenac and paracetamol was also effective in reducing the severity of post operative pain as compared to the control.

\section{Conclusion}

According to the finding and within the limitation of the study E-90 was effective in reducing the intensity of post operative pain compared to the other two groups statistically at $10 \%$ significance, when administerd in patients with irreversible pulpitis undergoing single visit root canal treatment, and movon-p was effective in reducing the post operative pain compared to the control group.

\section{References}

[1] Effects of three oral analgesics on post operative pain following root canal preparation : A controlled clinical trail. IEJ 45 76-82, 2012.

[2] Etoricoxib for arthritis and pain management TherClin Risk Manag. 2006 March; 2(1): 45-57.

[3] preclinical profile and comparison with other agents that selectively inhibit cyclooxygenase-2. J PharmacolExp Ther.2001;296:55866.

[4] Characterization of etoricoxib, a novel, selective COX-2 inhibitor. J ClinPharmacol. 2003;43:573-85.

[5] The COX-2 inhibitor etoricoxib did not alter the anti-platelet of low dose aspirin in health in healthy volunteers [abstract] Arthritis Rheum. 2001;44(Suppl 9):S135. 498

[6] Pharmacokinetics of etoricoxib in patients with renal impairment.JClinPharmacol. 2004 Jan;44(1):48-58

[7] To evaluate efficacy and safety of fixed dose combination of aceclofenac + paracetamol + thiocolchicoside (acenac-MR) in the treatment of acute low back pain. J Indian Med Assoc. 2012 Jan;110(1):56-8

[8] Treatment of chronic low back pain with etoricoxib, a new cyclo-oxygenase-2 selective inhibitor: improvement in pain and disability-a randomized, placebo-controlled, 3-month trial. J Pain. 2003 Aug;4(6):307-15

[9] COX-2 Selective Inhibitors in the Treatment of Arthritis: A Rheumatologist Perspective Current Topics in Medicinal Chemistry, Volume 5, Number 5 